\title{
Violence Detection With Two-Stream Neural Network Based on C3D
}

\author{
zanzan Lu, Minnan Normal University, China \\ Xuewen Xia, Minnan Normal University, China \\ (iD) https://orcid.org/0000-0002-4938-1479 \\ Hongrun Wu, Minnan Normal University, China \\ Chen Yang, School of Artificial Intelligence, Shenzhen Polytechnic, China
}

\begin{abstract}
In recent years, violence detection has gradually turned into an important research area in computer vision with many proposed models with high accuracy. However, there is unsatisfactory generalization ability of these methods over different datasets. In this paper, the authors propose a violence detection method based on C3D two-stream network for spatiotemporal features. First, the authors preprocess the video data of RGB stream and optical stream respectively. Second, the authors feed the data into two C3D networks to extract features from the RGB flow and the optical flow respectively. Third, the authors fuse the features extracted by the two networks to obtain a final prediction result. To testify to the performance of the proposed model, four different datasets (two public datasets and two self-built datasets) are selected in this paper. The experimental results show that the model has good generalization ability compared to state-of-the-art methods since it not only has good ability on large-scale datasets but also performs well on small-scale datasets.
\end{abstract}

\section{KEYWORDS}

C3D Network, Deep Learning, Feature Fusion, Spatiotemporal Features, Two-Stream Network, Video Detection, Violence Detection, Violent Datasets

\section{INTRODUCTION}

With the development and progress of society, a harmonious and stable social security becomes crucial. Thus, how to timely detect violence and then effectively reduce or prevent violent crimes become very important in now a days. Recently, various methods for feature extraction on images have been developed for a long time benefited from the rapid progress of computer technology, so extraction on images is relatively mature. For instance, in (LeCun, Bottou, Bengio, 1998), LeNet was firstly applied in handwritten number recognition, and ResNet (He, Zhang, et al, 2016) has recently gained popularity for image recognition. The accuracy of some image recognition methods has even surpassed that of humans. For example, Russakovsky et al. (Russakovsky, Deng, et al, 2015) evaluated human classification error is 5.1\% on a large-scale image dataset (ILSVRC2012-2014 classification test set), while the error of the ResNet model mentioned above was only $3.6 \%$ on the same dataset.

Unlike traditional image recognition, the violence detection, as a subclass of action detection, often occurs over a continuous period of time. Hence, an algorithm applied in the violence detection must take into account not only the spatial dimension of the image, but also the temporal dimension

\section{DOI: 10.4018/IJCINI.287601}

This article published as an Open Access article distributed under the terms of the Creative Commons Attribution License (http://creativecommons.org/licenses/by/4.0/) which permits unrestricted use, distribution, and production in any medium, provided the author of the original work and original publication source are properly credited. 
of the image. This makes it necessary for researchers to make their models capable of extracting spatiotemporal features simultaneously, as is the case in this paper. Based on the property, a number of models have been proposed in the last few decades (Ramzan, Abid, et al, 2019), such as two-stream network (Simonyan, Zisserman, 2014) and 3D Convolutional network (C3D) (Tran, Bourdev, et al, 2015; Ji, Xu, et al, 2012), etc. Each of these methods extracts the temporal and spatial information of the image in their own way, and yields its own characteristics.

In order to apply violence detection to real-world applications, it is necessary that the violence detection model has a favorable generalization capability. However, the generalization ability of existing models is still a challenge. For instance, unlike traditional machine learning, in order to obtain a promising generalization ability, majority of deep learning models need a large amount of data for training (Simonyan, Zisserman, 2014; Tran, Bourdev, et al, 2015), and the reality is that sometimes there is not that much data, which leads to unsatisfactory performance of the model. In order to solve this problem, researchers not only need to improve the model, but also to optimize the datasets.

Violence detection, as a subclass of action recognition, can use many of the methods of behavior recognition. Two-stream network detect action by combining temporal and spatial streams, an approach that has the advantage of being easily scalable and highly accurate. The C3D network uses the convolution of 3D structures for action recognition, which has the characteristics of structural simplicity and efficiency. Inspired by the excellent performance of two-stream network and C3D network in the field of action recognition, the authors tackle the challenges mentioned above by proposing a C3D-based two-stream network violence detection model.

The main contributions of this paper include:

1. Current mainstream methods are unable to learn effective models due to the lack of data on violence. Moreover, the existing violence detection datasets suffer from the problem of insufficient data quantity and single data scene. To tackle these problems, the authors collect violent videos from websites and process them. In this way, the authors create two new datasets in this work, i.e., public datasets and self-built datasets.

2. The authors find that previous models (e.g., C3D) did not perform very well on small-scale datasets, which indicates the low generalization ability of previous models on small-scale datasets. To further improve the generalization ability of the model, the authors improve the two-stream network so that authors' C3D two-stream network can not only perform well on large-scale datasets, but also achieve high accuracy on small-scale datasets.

The remaining parts of the paper is organized as the following. Section briefly describes related work in the area of violence detection. The detail of the proposed model is introduced in Section 3, while the detail of two types of datasets are described in section 4. Section 5 contains the results and discussion of the experiment and the conclusion and future work are presented in section 6 .

\section{Related Work}

Violence detection, as a hot topic in computer vision applications, is increasingly sought after by researchers. Today, many techniques have emerged in the field of violence detection, and some have even been applied in the real world (Akt1, Tataroğlu, Ekenel, 2019; Febin, Jayasree, Joy, 2019; Roshan, Srivathsan, et al, 2020; Singh, Patil, Omkar, 2018). Generally, these techniques fall into two main categories: the traditional machine learning and the recently proposed deep learning.

In the early days, researchers generally used the traditional machine learning methods to extract features of violent videos. For instance, Clarin et al. (Clarin, Dionisio, et al, 2005) suggested a motion intensity analysis on skin and blood to detect movie violence. Similarly, Chen et al. (Chen, Hsu, et al, 2011) integrated facial, blood and movement information to determine if the action scene has violent content. The main contribution of the work is applying the presence of highly relevant objects 
(e.g., gunshots, flames, blood, explosions) to identify violent incidents. Researchers later began to consider combining audio and video to improve the identify accuracy. For example, Mahadevan et al. (Mahadevan, Bhalodia, Vasconcelos, 2010) developed a system that can identify violent behavior by the degree of motion and sound combined with the characteristics of blood and flame. Furthermore, some researchers are beginning to extract features by combining the temporal and spatial aspects of the video. For instance, Zhou et al. (Zhou, Ding, et al, 2018) leveraged Local Histogram of Oriented Gradient (LHOG) and Local Histogram of Optical Flow (LHOF) methods to extract features from RGB images and optical flow images, respectively, to classify violent videos. Besides, the researchers attempt to create some video descriptors to feed to the linear classifier to make it more accurate. Therefore, many classical approaches have been proposed: such as violent flow descriptors (ViF) (Hassner, Itcher, Kliper-Gross, 2012), oriented violent flow descriptor (OViF) (Gao, Liu, et al, 2016), improved dense trajectories (iDT) (Wang, Schmid, 2013), statistical characteristics of the optical flow (SCOF) (Huang, Chen, 2014).

In recent years, the progress of deep learning technology driven by the rapidly development of computer hardware technology enables the automatic violence detection becomes feasible. Compared with traditional machine learning, the recently proposed deep learning has the advantages of a high adaption ability and a high accuracy rate. Therefore, numerous methods are developed based on the deep learning. For example, Wu et al. (Wu, Liu, et al, 2020) integrated audio and video characteristics to identify violent behavior. Compare with combining sound and video, integrating the temporal and spatial features of video has attracted more attention of researchers in recent years. For instance, Simonyan et al. (Simonyan, Zisserman, 2014) trained the Convolutional Neural Network (CNN) model on RGB images flow and dense optical flow, and then merged the class scores of the two networks intending to obtain a better classification result. Based on the approach of two-stream network, Feichtenhofer et al. (Feichtenhofer, Pinz, Zisserman, 2016) considered using the CNN for spatial as well as temporal convergence, while replacing the CNN with the VGG-16 network. These methods are based on improvements in two-stream network, which are also applicable to violence detection. For instance, Xia et al. (Xia, Zhang, et al, 2018) used CNN training to obtain both appearance representation and motion representation, and then used support vector machine(SVM)(Cortes, Vapnik, 1995) to classify and fuse the labels. Another popular branch is using C3D (Tran, Bourdev, et al, 2015) for spatiotemporal feature extraction. Researchers use C3D for violence detection, such as Ullah et al. (Ullah, Muhammad, 2019), Song et al. (Song, Zhang, et al, 2019), Ding et al. (Ding, Fan, et al, 2014), Accattoli et al. (Accattoli, Sernani, et al, 2020). In addition to the aforementioned work, researchers have also focused on Graph Convolutional Network (GCN) for the detection of abnormal behavior. For instance, Yan et al. (Yan, Xiong, Lin, 2018) extended the GCN to the spatiotemporal domain and called it the Spatiotemporal Graph Convolutional Network (ST-GCN). Based on GCN, Si et al. (Si, Chen, et al, 2019) proposed a novel attention-enhancing graph convolutional LSTM network (AGCLSTM) for human action recognition from skeletal data.

To show the related work more visually, the authors organize them into a table, as shown in Table 1. In the table, we can see that researchers have been constantly exploring new methods for violence detection. Among these models, traditional machine learning models require manual extraction of features appear tedious, while deep learning models do not perform very well on small-scale datasets, so the authors want to build a deep learning model that can have good generalization ability on smallscale datasets, and this model is efficient.

\section{C3D Two-stream Network}

As shown in Fig. 1, the proposed C3D two-stream network is consisted of three main modules, i.e., the RGB channel, the optical flow channel, and the feature fusion. The first two modules adopt C3D structure. Since the output of RGB channel and the optical flow channel have consistent structures, the authors use the concatenate method to fuse them. 
Table 1. Summary of related work.

\begin{tabular}{|c|c|c|c|}
\hline Type & Author & Date & Brief Description \\
\hline \multirow[t]{8}{*}{ Machine Learning } & Clarin et al. & 2005 & Analysis of the intensity of movement of the skin and blood \\
\hline & Chen et al. & 2011 & Combining facial, blood and motion information. \\
\hline & Mahadevan et al. & 2010 & $\begin{array}{l}\text { Using the degree of motion and sound combined with the } \\
\text { characteristics of blood and flame }\end{array}$ \\
\hline & Zhou et al. & 2018 & $\begin{array}{l}\text { Features are extracted from RGB images and optical } \\
\text { flow images using Local Histogram of Oriented Gradient } \\
\text { (LHOG) and Local Histogram of Optical Flow (LHOF) }\end{array}$ \\
\hline & Hassner et al. & 2012 & Violent flow descriptors (ViF) \\
\hline & Gao et al. & 2016 & Oriented violent flow descriptor $(\mathrm{OViF})$ \\
\hline & Wang et al. & 2013 & Improved dense trajectories (iDT) \\
\hline & Huang et al. & 2014 & Statistical characteristics of the optical flow (SCOF) \\
\hline \multirow[t]{11}{*}{ Deep Learning } & Wu et al. & 2020 & Integrated audio and video features \\
\hline & Simonyan et al. & 2014 & Two-Stream network \\
\hline & Feichtenhofer et al. & 2016 & $\begin{array}{l}\text { Based on Two-Stream network, but using VGG-16 network } \\
\text { to replace CNN }\end{array}$ \\
\hline & Xia et al. & 2018 & $\begin{array}{l}\text { Based on Two-Stream network, but classified with support } \\
\text { vector machine (SVM) }\end{array}$ \\
\hline & Tran et al. & 2015 & 3D Convolutional network (C3D) \\
\hline & Ullah et al. & 2019 & \multirow[t]{4}{*}{ C3D-based violence detection } \\
\hline & Song et al. & 2019 & \\
\hline & Ding et al. & 2014 & \\
\hline & Accattoli et al. & 2020 & \\
\hline & Yan et al. & 2018 & Spatiotemporal Graph Convolutional Network (ST-GCN) \\
\hline & Si et al. & 2019 & $\begin{array}{l}\text { Attention-enhancing graph convolutional LSTM network } \\
\text { (AGCLSTM) }\end{array}$ \\
\hline
\end{tabular}

Figure 1. The Framework of C3D Two-stream Network.

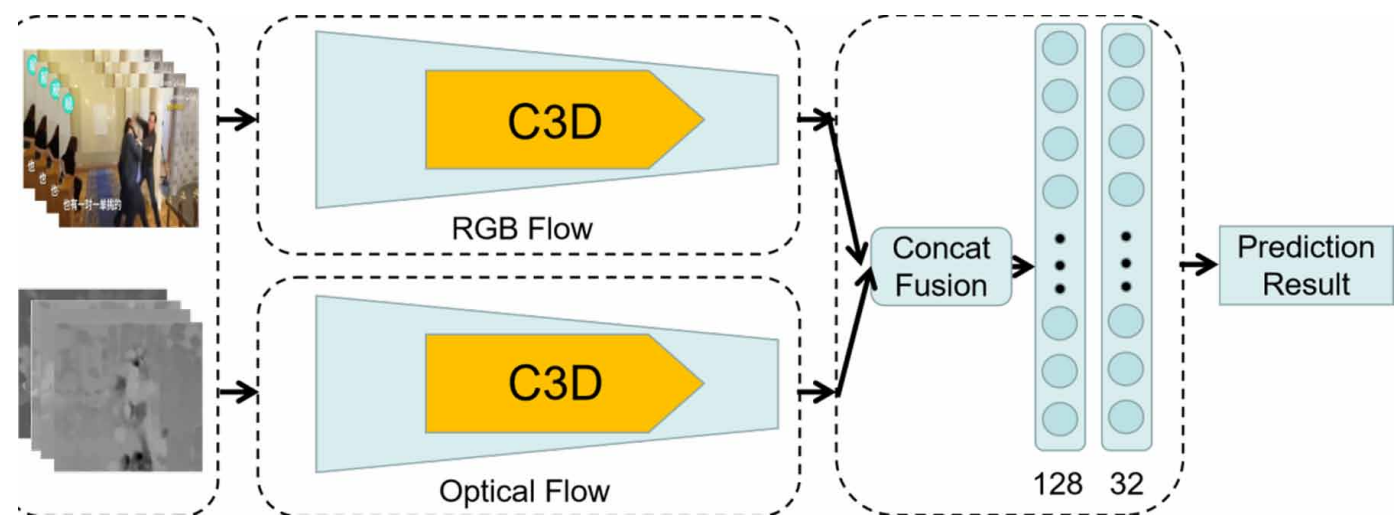


Figure 2. $C 3 D$ two-stream network architecture (single stream) for RGB/OPT stream. Here, all $3 D$ convolutional kernels are $3 \times 3 \times 3$ with stride 1 , and all pooling kernels are $2 \times 2 \times 2$, except for the first pooling layer which is $1 \times 2 \times 2$.

C3D architecture

\begin{tabular}{|c|c|c|c|c|c|c|c|c|c|c|c|c|c|c|c|}
\hline $\begin{array}{c}\text { ConvA1 } \\
64\end{array}$ & $\overline{0}$ & $\begin{array}{c}\text { ConvB1 } \\
128\end{array}$ & $\overline{0}$ & $\begin{array}{c}\text { ConvC1 } \\
256\end{array}$ & $\begin{array}{c}\text { ConvC2 } \\
256\end{array}$ & ס & $\begin{array}{c}\text { ConvD1 } \\
512\end{array}$ & $\begin{array}{c}\text { ConvD2 } \\
512\end{array}$ & ס & $\begin{array}{c}\text { ConvE1 } \\
512\end{array}$ & $\begin{array}{c}\text { ConvE2 } \\
512\end{array}$ & $\overline{0}$ & $\begin{array}{c}\mathrm{fc} \\
4096\end{array}$ & $\begin{array}{c}\text { fc } \\
4096\end{array}$ & 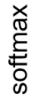 \\
\hline
\end{tabular}

C3D Two-Stream network architecture (single stream)

Replace

\begin{tabular}{|c|c|c|c|c|c|c|c|c|c|c|c|c|c|c|c|}
\hline $\begin{array}{c}\text { ConvA1 } \\
64\end{array}$ & ס & $\begin{array}{c}\text { ConvB1 } \\
128\end{array}$ & $\overline{0}$ & $\begin{array}{c}\text { ConvC1 } \\
256\end{array}$ & $\begin{array}{c}\text { ConvC2 } \\
256\end{array}$ & : & $\begin{array}{c}\text { ConvD1 } \\
512\end{array}$ & $\begin{array}{c}\text { ConvD2 } \\
512\end{array}$ & $\overline{0}$ & $\begin{array}{c}\text { ConvE1 } \\
512\end{array}$ & $\begin{array}{c}\text { ConvE2 } \\
512\end{array}$ & $\begin{array}{l}\overline{0} \\
0 \\
0\end{array}$ & $\begin{array}{c}\text { fc } \\
4096\end{array}$ & $\begin{array}{c}\text { fc } \\
4096\end{array}$ & $\begin{array}{l}\text { fc } \\
10\end{array}$ \\
\hline
\end{tabular}

Violence detection in this study means recognizing violent scenes from videos, so the temporal scale is also a valuable cue for the characterization of the complex motion patterns. 3D ConvNet is well-suited for spatiotemporal feature learning owing to 3D convolution and 3D pooling operations. Compared to the 2D ConvNet, convolution and pooling operations are performed spatiotemporal in 3D ConvNet, while in 2D ConvNet they are done only spatially. Thus, in this study, the 3D ConvNet is adopted to extract spatiotemporal features from both RGB flow and optical flow.

In this work, the authors make an improvement for the network structure of the 3D ConvNets. Unlike the traditional single-stream C3D ConvNets, in which a softmax layer is a fully connected layer. The reason for this is that the original C3D network is outputted after fc4096 layer, but in the C3D two-stream network the authors need to fuse the features after the single stream before outputting them. The new network architecture proposed by us has 8 convolutional, 5 max-pooling layers and 3 fully connected layers. The detail of the network architecture is shown in Fig. 2.

One of the most distinctive features in two-stream network is the merge layer, and the method of feature fusion varies from network to network. In Fig. 3, the authors show the structure of the merge layer at the end of two C3D networks using the concatenation fusion method. Both the RGB flow and the optical flow pass through C3D network, so that the dimensionality of the features maps becomes identical. Then, the authors combine the two output feature maps into the new feature map at the merge layer.

Finally, the new feature maps pass through the FC layer and then output the prediction results. To further improve the accuracy of the network, the authors try to compare several fusion methods and finally decide to use concatenation fusion. Concatenation fusion, $Y=f^{\text {concat }}\left(X^{A}, X^{B}\right)$, is a very simple but effective method. It stacks two feature vectors along the channel dimension to get the final output. The fusion can be defined as the follows.

$\mathbf{Y}=\mathbf{X}_{c}^{A} \cup \mathbf{X}_{d}^{B}$

where $\mathbf{X}_{c}^{A}$ denotes the A stream input containing c channels, and $\mathbf{X}_{d}^{B}$ denotes the B stream input containing d channels.

After the fusion layer the authors then use two FC layers for processing. At the end of the model, the authors use a sigmoid layer to make predictions about the results.

\section{Introduction to Datasets}

In the experiments the authors use four violent datasets, including two public datasets (Hockey Fight and Surveillance Cameras) and two new datasets (HK-10000 and BL-2314). As the small data scale 
Figure 3. The structure of the concatenation fusion.

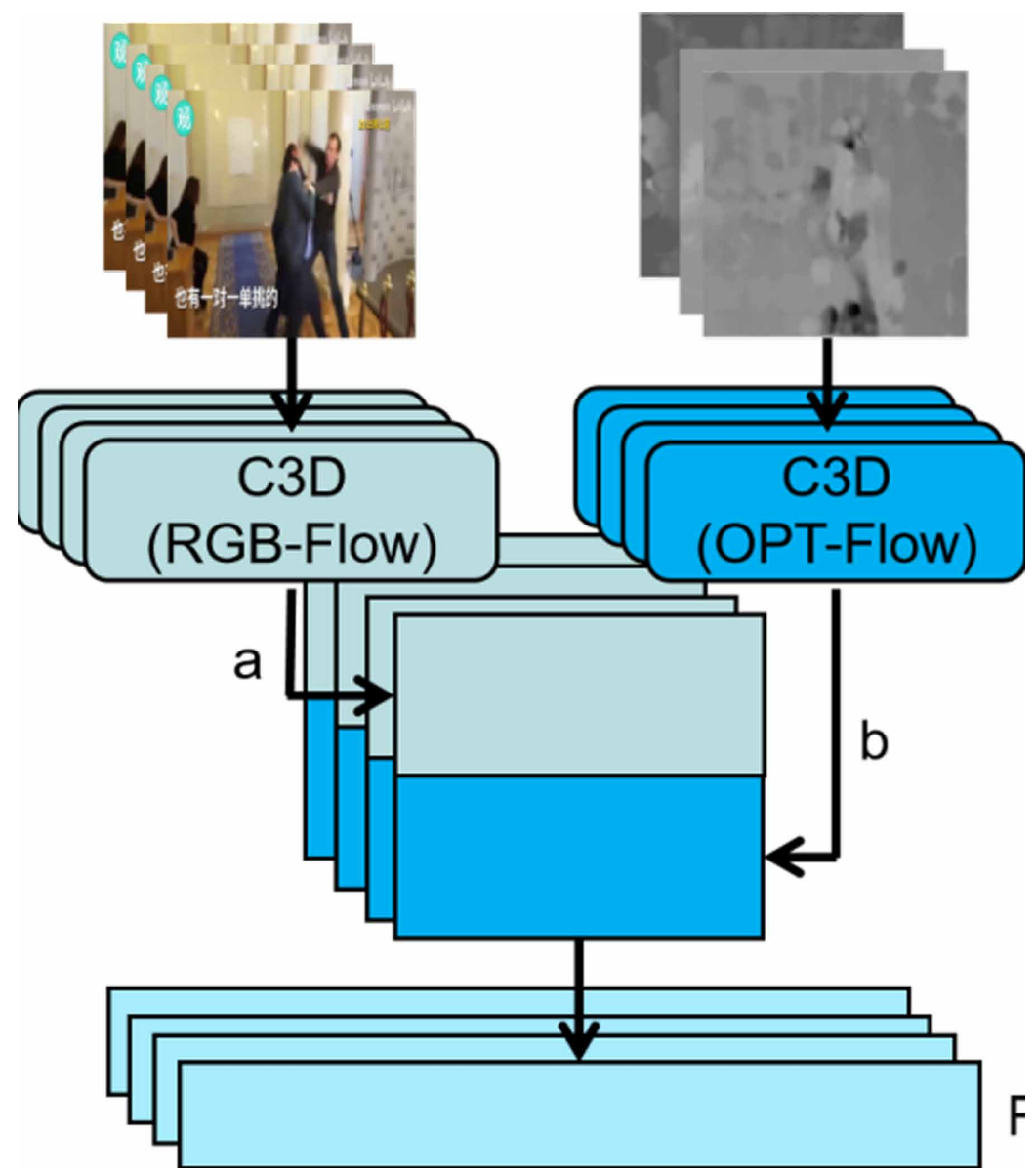

of Hockey Fight and Surveillance Cameras, these two datasets are frequently used to evaluate the effectiveness of violence-detection methods. Though many violence datasets have been published in last few years, they still suffer from small size, single scenes, poor resolution, and blurring, etc. To address these problems, the authors introduced two new datasets ${ }^{1}$, i.e., BL-2314 and HK-10000, in authors' study. The BL-2314 consists of many different fighting scenarios, and the size of HK10000 dataset even reaches to 10,000 videos. The authors hope that these new datasets can provide convenience and assistance to researchers in their violence detection research work.

The authors' reasons for choosing these four datasets are as follows:

1. They cover a variety of scenarios, including multi-player fights and two-player fights.

2. They have variations in frame rate, resolution, etc.

3. They have different sizes of data.

Thus the experiments on these datasets allow a more comprehensive validation of the performance of the authors' model.

By the way, Collecting data to build a dataset is a very tedious task, so we use a web crawler program to obtain videos related to the keyword "violence" from some video websites, and then manually edit, filter and classify these videos. 
Figure 4 shows some video samples from the four datasets, and the attributions of the data scale, including frame rate, scenario, length, and annotation type of videos, are summarized in Table 2.

Table 2. Information of sample for each dataset.

\begin{tabular}{|c|c|c|c|c|c|}
\hline Dataset & Data Scale & Frame Rate & Scenario & Violent & Non-Violent \\
\hline Surveillance & 300 & Variable & Multiple & 150 & 150 \\
\hline Hockey & 1000 & 25 & Single & 500 & 500 \\
\hline BL-2314 & 2314 & Variable & Multiple & 2314 & 0 \\
\hline HK-10000 & 10000 & 25 & Single & 5000 & 5000 \\
\hline
\end{tabular}

Figure 4. Sample video frames randomly selected from: (A) BL-2314, (B) HK-10000, (C) Hockey Fight and (D) Surveillance Cameras

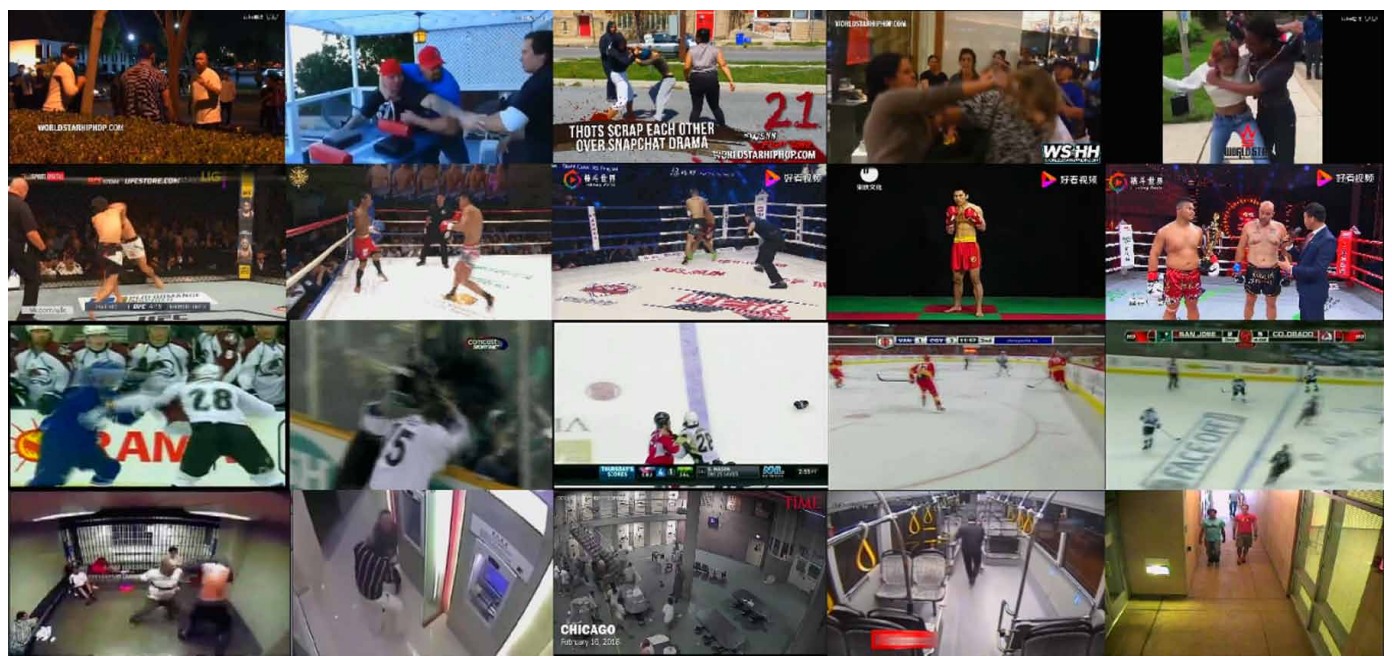

The details of the two new datasets and two widely used datasets are described as follows:

Hockey: Nievas et al. presented the Hockey Fight dataset in (Nievas, Suarez, et al, 2011). The Hockey dataset includes 1,000 clips captured in hockey games of the National Hockey League, and half of them (500 clips) are labeled as fight and another half as non-fight.

Surveillance: Akt1 et al. collected 300 fight scenes in total from surveillance camera videos available at YouTube (Aktı, Tataroğlu, Ekenel, 2019). The surveillance dataset includes various types of fight scenarios such as kick, fist, and hitting with an object. There are 150 fight sequences and 150 non-fight sequences in the dataset, and the videos have different sizes and different number of frames.

HK-10000: The authors collected a boxing match dataset from Haokan ${ }^{2}$, in which all the videos are taken in a single boxing match scene. This dataset has 10,000 videos, and each video is edited to 3 seconds and its frame rate is 25 frames per second. The authors divide the dataset into two categories, i.e. 5,000 violent video clips and 5,000 non-violent video clips. In this dataset, the resolution of videos are different, such as $640 \times 360$ pixels and $1280 \times 720$ pixels. 
BL-2314: This dataset is collected from bilibili ${ }^{3}$, and it is consisted of two-person and multi-person fighting scenes. It is noticeable that some of the videos are filmed using smart phones and some are extracted from surveillance cameras. So, for the videos in BL-2314, the settings of their resolution are different (such as $320 \times 240,1280 \times 720,1440 \times 720,1920 \times 1080$, etc.), and their frame rates vary from 12 to 30 . In addition, the fighting situation between people in the video are various, for example pushing, kicking, and street fights.

\section{EXPERIMENTS AND DISCUSSION}

In this section, the authors first describe the parameters settings of their model, and then introduce three experiments to evaluate their proposed model by using the four datasets.

\section{Experimental Setup}

To further enhance the model data training, the authors first preprocess the video data and then train the model.

First, the authors need to preprocess the video. Fig. 5 shows the procedure of data preprocessing. The authors extract each frame from the input video, and then divide it into two parts, i.e., a RGB flow and an optical flow. For the RGB flow, the authors reshape each image frame into a matrix with size $224 \times 224 \times 3$. In terms of the optical flow, the authors first transform each frame into a grayscale image and then reshape it into size $224 \times 224 \times 1$, and then extract the horizontal (x-axis) and vertical (y-axis) features from optical flow to obtain two matrices $224 \times 224 \times 1$. After that, the $x$-axis and $y$-axis matrices are combined into a new matrix with size $224 \times 224 \times 2$. Finally, by combing the optical flow and the RGB flow, the size of the obtained matrix is $224 \times 224 \times 5$.

After the data preprocessing, a $224 \times 224 \times 5$ matrix can be obtained for the training process. During the data training process, the optimization objective is defined on the video-level predictions, and combining standard categorical cross-entropy loss, the loss function is defined as follows,

$$
H(p, q)=-\sum_{i=1}^{n} p\left(x_{i}\right) \log \left(q\left(x_{i}\right)\right)
$$

where $p\left(x_{i}\right)$ is the ground truth of the $\mathrm{i}^{\text {th }}$ sample; $q\left(x_{i}\right)$ is the predicted probability of the $\mathrm{i}^{\text {th }}$ sample.

In this paper, the proposed model is optimized by using back propagation with stochastic gradient descent (SGD) to update the model parameters. The authors set the momentum of SGD as 0.9, and the learning rate decay as 1e-6.

All experiments are run on a workstation with a 10-core Intel Xeon CPU (E5-2660 v3, 2.60 GHz), 256GB RAM, and a NVIDIA Tesla V100 GPU.

\section{Comparison with State-of-the-art Methods}

The authors compare the proposed model with three machine learning approaches (i.e., SIFT+SVM, HOG+SVM, HOG+RF) and three deep learning approaches (i.e., ConvLSTM, C3D, I3D), which are usually used to detect violence scenes in current studies.

SIFT+SVM uses SVM (Cortes, Vapnik, 1995) for classification after extracting features with scale-invariant feature transform (SIFT) (Lowe, 2004).

In HOG+SVM, Histogram of Oriented Gradient (HOG) (Dalal, Triggs, 2005) is selected to extracting features, which SVM is adopted for classification. 
Figure 5. The diagram of data preprocessing. The steps of data preprocessing are as follows: 1 . Transform each frame of the input video into two parts: optical flow and RGB flow; 2. RGB flow is reshaped to a $224 \times 224 \times 3$ matrix; optical flow is transformed to a grayscale image first, then it reshape into two matrices of $224 \times 224 \times 1 ; 3$. The authors extract the horizontal (x-axis) and vertical (y-axis) features from optical flow to obtain two matrices with $224 \times 224 \times 1$, then combine this two matrices into a new matrix with size $224 \times 224 \times 2$; 4 . Combined the matrices of optical flow and RGB flow with a $224 \times 224 \times 5$ matrix.

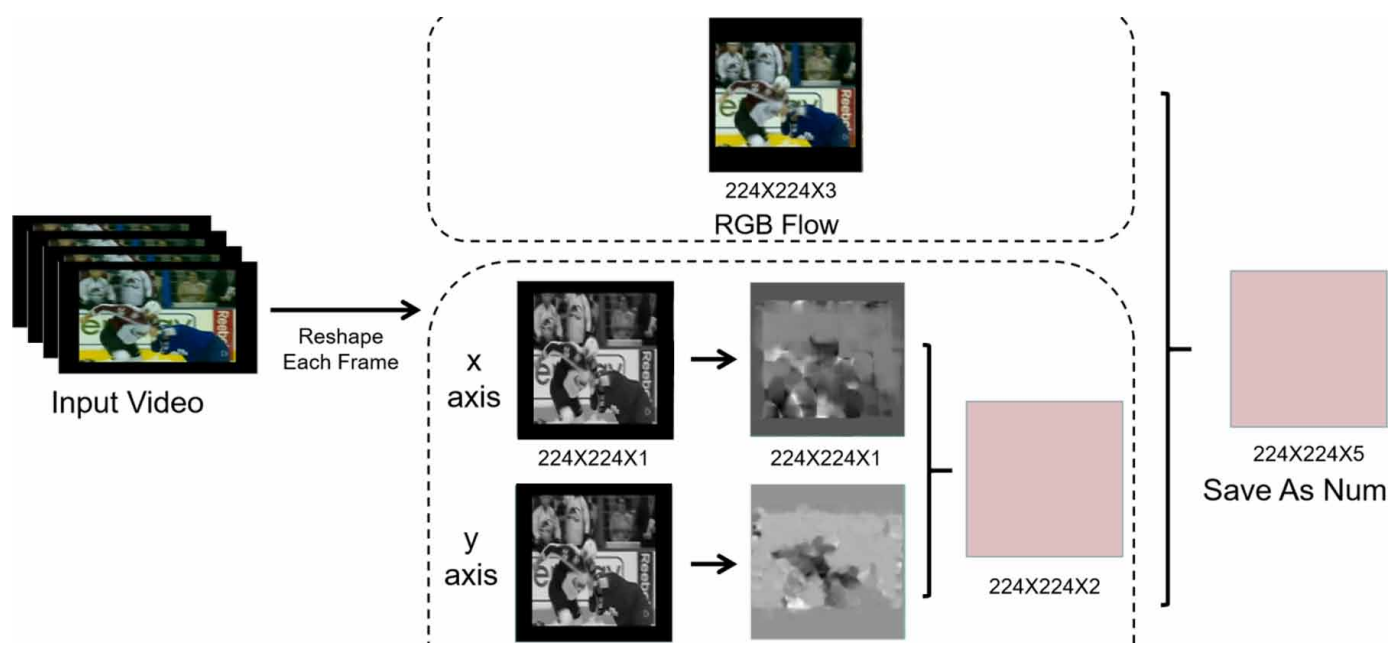

In HOG+RF, HOG is used as an extractor of features and Random Forest (RF) (Breiman, 2001) is used as a classifier.

ConvLSTM (Donahue, Anne Hendricks, et al, 2015) is a network model that combines 2D convolutional network with LSTM.

C3D (Tran, Bourdev, et al, 2015) network extends the 2D convolutional network to 3D in order to extract temporal features.

I3D (Carreira, Zisserman, 2017) is a two-stream network based on an inflated 3D network.

Table 3 presents the accuracy of the proposed C3D two-stream network and the six approaches in the four datasets with an 80:20 split of training and testing sets. These four datasets have different sizes, where Surveillance has 300 videos, Hockey contains 1000 videos, BL-2314 owns 2314 videos, and HK-10000 includes 10000 videos. Among them, because the 2314 videos contained in BL-2314 are all violence video clips, we randomly select 2314 non-violence video clips from the other three datasets as its non-violence video part, so that the BL-2314 dataset has a total of 4628 video clips.

As can be seen from the Table 3, the machine learning (ML) approaches are slightly better than the deep learning approaches when the data scale is small, but compared to other deep learning approaches the proposed C3D two-stream network performs the best in small scale data. SIFT+SVM, which is a popular ML approach, achieves the highest detection accuracy (88.22\%) on Surveillance who has the smallest date scale. In addition, the accuracy of proposed C3D two-stream network is $69.17 \%$, which is higher than that of the other three deep learning (DL) approaches on the Surveillance dataset. For the datasets Hockey and HK-10000 who have the largest data scale, the detection results of DL approaches are more accurate than that of ML approaches, and the proposed C3D gets the best detection results. The reason of the results is that in DL, the larger the amount of data the better it is to cover all the key features. On the contrary only a lot of a priori knowledge is used at the beginning in ML.

However, for the detection results on the dataset BL-2314, the ML approaches obtain better performance than the DL approaches. Concretely, HOG+RF obtains the highest accuracy $(91.84 \%)$ in the ML and DL approaches, while the accuracy of the C3D two-stream network is $78.30 \%$, which 
Table 3. Comparison of the accuracy of the proposed method with other methods on different datasets, where Ours(avg) is the average of the results of training the RGB and optical streams separately and then averaging their predictions according to I3D's method.

\begin{tabular}{|c|c|c|c|c|c|}
\hline Type & Method & Surveillance & Hockey & BL-2314 & HK-10000 \\
\hline \multirow{3}{*}{$\begin{array}{c}\text { Machine } \\
\text { Learning }\end{array}$} & SIFT+SVM & $\mathbf{8 8 . 2 2 \%}$ & $62.62 \%$ & $82.91 \%$ & $76.20 \%$ \\
\cline { 2 - 6 } & HOG+SVM & $67.67 \%$ & $71.41 \%$ & $78.68 \%$ & $65.52 \%$ \\
\cline { 2 - 6 } & HOG+RF & $79.65 \%$ & $76.01 \%$ & $\mathbf{9 1 . 8 4 \%}$ & $79.46 \%$ \\
\hline \multirow{3}{*}{$\begin{array}{c}\text { Deep } \\
\text { Learning }\end{array}$} & ConvLSTM & $56.67 \%$ & $89.50 \%$ & $72.51 \%$ & $86.00 \%$ \\
\cline { 2 - 6 } & C3D & $43.33 \%$ & $93.50 \%$ & $75.00 \%$ & $81.10 \%$ \\
\cline { 2 - 6 } & I3D(Fusion) & $56.67 \%$ & $89.50 \%$ & $74.19 \%$ & $\mathbf{8 6 . 7 3 \%}$ \\
\cline { 2 - 6 } & Ours & $69.17 \%$ & $\mathbf{9 6 . 5 0 \%}$ & $78.30 \%$ & $84.20 \%$ \\
\cline { 2 - 6 } & Ours(avg) & $51.67 \%$ & $83.00 \%$ & $63.58 \%$ & $78.58 \%$ \\
\hline
\end{tabular}

is higher than the other three DL approaches. This may be due to the fact that multi-scene datasets have more different features than single-scene datasets.

In Fig. 6, the authors present the relationship between epoch (the total number of iterations) and detection accuracy for the five DL methods. The plots show that the change range of the accuracy is very small (tending to 0) after 60 epochs, so the authors set the epoch number to 60 for the DL approaches. Also, we can see that authors' model outperforms other DL models on the Surveillance and Hockey datasets.

Finally, the authors also compare the efficiency of these deep learning models running for 60 epochs. The results are shown in the Table 4, it can be seen that the two-stream C3D is second only to the single-stream C3D in terms of running speed, much faster than ConvLSTM and I3D.

\section{Comparison of Proportional Sensitivity of Datasets}

The authors analyze the performance of the proposed model with different train-test splits. In Table 5, three train-test ratios, i.e. 4:6, 6:4 and 8:2, are selected. The authors choose the train-test ratios in this way because these ratios are relatively common. For example, Accattoli et al. (Accattoli, Sernani, et al, 2020) use a 5-fold cross validation scheme (the train-test ratio is 8:2). Note that all the other parameters in this experiment, such as learning rate and epoch etc., remain unchanged. The results show that the C3D two-stream network attains the best performance on the Surveillance, Hockey and HK-10000 when the train-test ratio is 8:2. On the contrary, the detection accuracy of the proposed model reaches to $99.41 \%$ on the BL-2314 dataset with 6:4 split. This may be due to the fact that the two single streams in the C3D two-stream network model have different desires for the amount of data. For example, on small-scale datasets (e.g., Surveillance), the model is more hungry for data, when more testing data can improve the accuracy of the model. In contrast for larger datasets (e.g., BL-2314) too much training data can reduce its accuracy.

Fig. 7 shows the detection accuracy at different epoch on the two datasets with different traintest splits. Note that, due to the space limitation, only one small-scale dataset (Surveillance) and one large-scale dataset (BL-2314) are selected in the experiment. We can see that the accuracy of the C3D two-stream network increases with the amount of training data on Surveillance who is a small-scale dataset. However, in the large-scale datasets, i.e., BL-2314, the accuracy of the model reaches its maximum when the amount of training data is small and decreases when the number of training is too large. 
Figure 6. Comparison of different methods on the Surveillance dataset and Hockey dataset. (a) shows the val accuracy of these different methods on Surveillance dataset for 60 epochs. (b) shows the val accuracy of these different methods on Hockey dataset for 60 epochs.
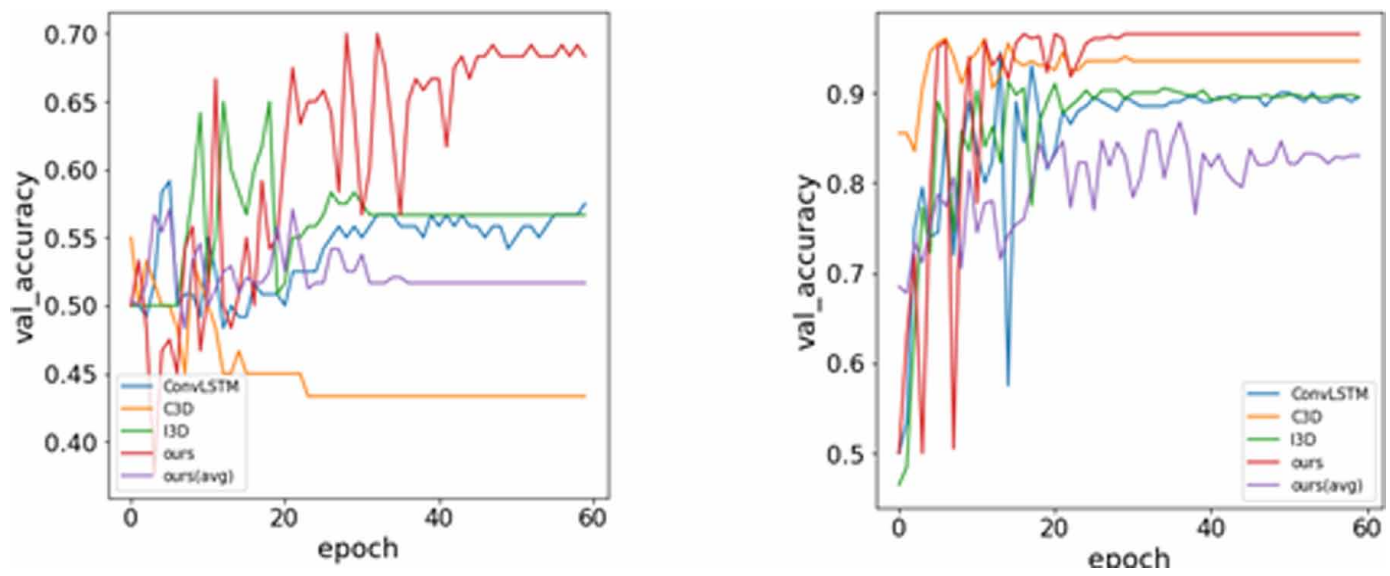

\section{Comparing the impact of RGB-Stream and OPT-Stream}

To further investigate the effect of RGB-Stream module and OPT-Stream module on the results of C3D two-stream network, the authors trained the optical and RGB stream in the two-stream C3D network on four different datasets. In this experiment, the authors make some adjustments to the C3D two-stream network by separately using the RGB stream and optical stream from the C3D two-stream network for training, and then outputting the classification results directly without feature fusion. The experimental results are demonstrated in Table 6, in which the train-test ratio of the dataset is set as $8: 2$ and all other parameters are the same as above.

From Table 6 we can observe that OPT-Stream outperforms RGB-Stream when the dataset size is small. On the contrary, RGB-Stream dominates OPT-Stream on the large-scale dataset. To

Table 4. Comparison of the time spent by the four deep learning models.

\begin{tabular}{|c|c|c|c|c|}
\hline \multirow{2}{*}{ Datasets } & \multicolumn{4}{|c|}{ Average Time Spent (s) / Epoch } \\
\cline { 2 - 5 } & $\begin{array}{c}\text { ConvLSTM } \\
\text { (Single Stream) }\end{array}$ & $\begin{array}{c}\text { C3D } \\
\text { (Single Stream) }\end{array}$ & $\begin{array}{c}\text { I3D } \\
\text { (Two Stream) }\end{array}$ & $\begin{array}{c}\text { Ours } \\
\text { (Two Stream) }\end{array}$ \\
\hline Surveillance & 65 & 40 & 110 & 93 \\
\hline Hockey & 229 & 147 & 370 & 326 \\
\hline BL-2314 & 1063 & 738 & 1760 & 1487 \\
\hline HK-10000 & 2320 & 1596 & 3828 & 3392 \\
\hline
\end{tabular}

Table 5. Comparison of the accuracy of the proposed method with different train-test ratios on different datasets.

\begin{tabular}{|c|c|c|c|c|}
\hline Training:Validation & Surveillance & Hockey & BL-2314 & HK-10000 \\
\hline $4: 6$ & $48.89 \%$ & $90.75 \%$ & $99.35 \%$ & $79.90 \%$ \\
\hline $6: 4$ & $54.58 \%$ & $90.25 \%$ & $99.41 \%$ & $79.45 \%$ \\
\hline $8: 2$ & $69.17 \%$ & $96.50 \%$ & $78.30 \%$ & $84.20 \%$ \\
\hline
\end{tabular}


Figure 7. The accuracy comparison experiments of the C3D two-stream network on the Surveillance and BL-2314 datasets with different training and testing ratios, respectively. (a) is a line graph of accuracy by training the C3D two-stream network on the Surveillance dataset in the ratio of 4:6, 6:4 and 8:2 with 60 epoch, respectively. (b) is a C3D two-stream network on the BL-2314 dataset also in the ratio of $4: 6,6: 4$ and $8: 2$ for the train-test ratio.
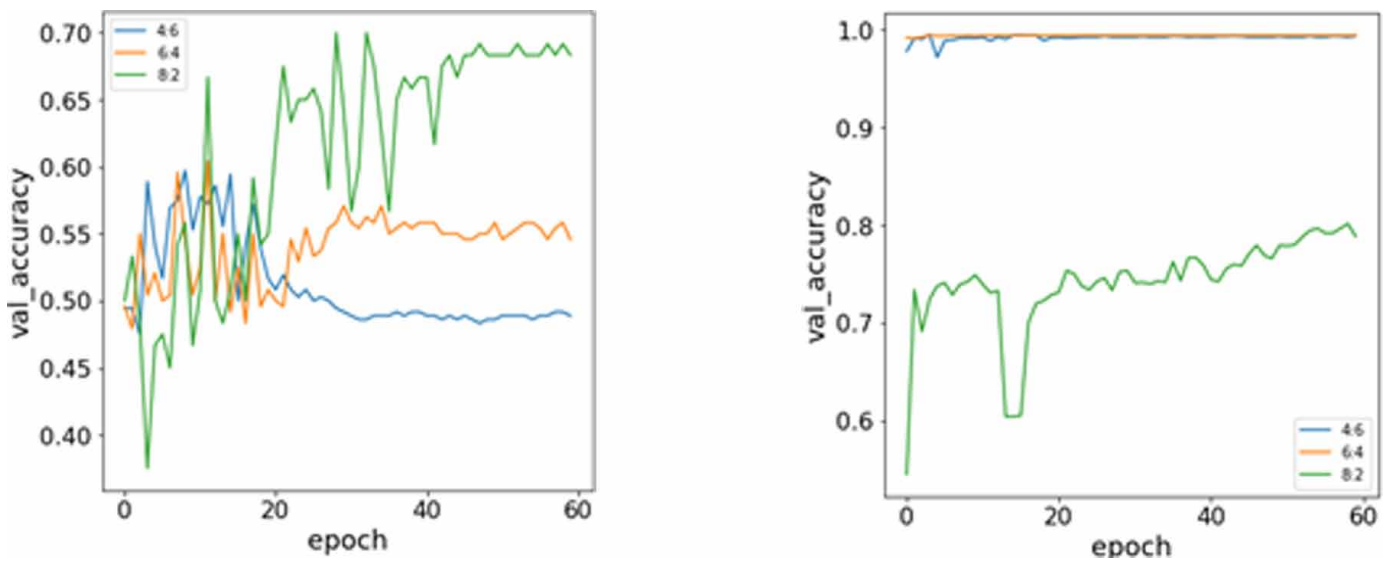

Table 6. Comparison of the accuracy of the proposed method with RGB stream and optical stream on different datasets.

\begin{tabular}{|c|c|c|c|c|}
\hline Stream & Surveillance & Hockey & BL-2314 & HK-10000 \\
\hline RGB & $43.33 \%$ & $93.50 \%$ & $75.00 \%$ & $81.10 \%$ \\
\hline OPT & $60.00 \%$ & $72.50 \%$ & $52.16 \%$ & $76.05 \%$ \\
\hline
\end{tabular}

further analyze the performance of OPT-Stream and RGB-Stream in C3D two-stream network in each epoch, the authors compare the accuracy of these two streams in 60 epochs, the results of which are demonstrated by Fig. 8. The results in Fig. 8 manifest that in the small-scale dataset, the optical stream performs better than the RGB stream, while in the large-scale dataset, the RGB stream displays favorable results than the optical stream. This can explain why the excessive amount of training data in the previous experiment led to the decline of model's effect. This is due to the fact that optical stream has invariance to the appearance of the image and therefore has an advantage when the data size is small. However, when the data scale is large, RGB stream is able to adequately cover key features of the image, which makes the invariance of the optical stream less important. Therefore, the C3D two-stream network can take full advantage of optical stream on small-scale datasets and can compensate for the lack of optical stream with RGB stream on large-scale datasets, which makes the C3D two-stream network have good generalization ability on small-scale and large-scale datasets.

\section{Conclusion and Future Work}

In this paper, the authors propose a new C3D two-stream model for violence detection based on spatiotemporal features and also provide two large-scale violence datasets. First the authors use the improved C3D network to extract spatial and temporal features of optical flow and RGB flow. Then, the authors use concatenation fusion to fuse the optical flow and RGB flow. Finally, the authors pass the fused features to the sigmoid layer to obtain the final classification results. To validate the performance of this model, a wealth of experiments between C3D two-stream model and other selected six models are conducted on four different datasets. The experimental results indicate that the C3D two-stream network works very well on both small datasets and large-scale datasets, so its generalization ability can be well applied in the real world. Moreover, two experiments, i.e., the 
Figure 8. Comparison of separately train optical stream and RGB stream on C3D two-stream model. (a) is a comparison of the effect of optical and RGB streams on the Surveillance dataset. (b) compares the effect of optical and RGB streams on the Hockey dataset. (c) is a comparison of the effect of optical and RGB streams on the BL-2314 dataset. (d) compares the effect of optical and RGB streams on the HK-10000 dataset.
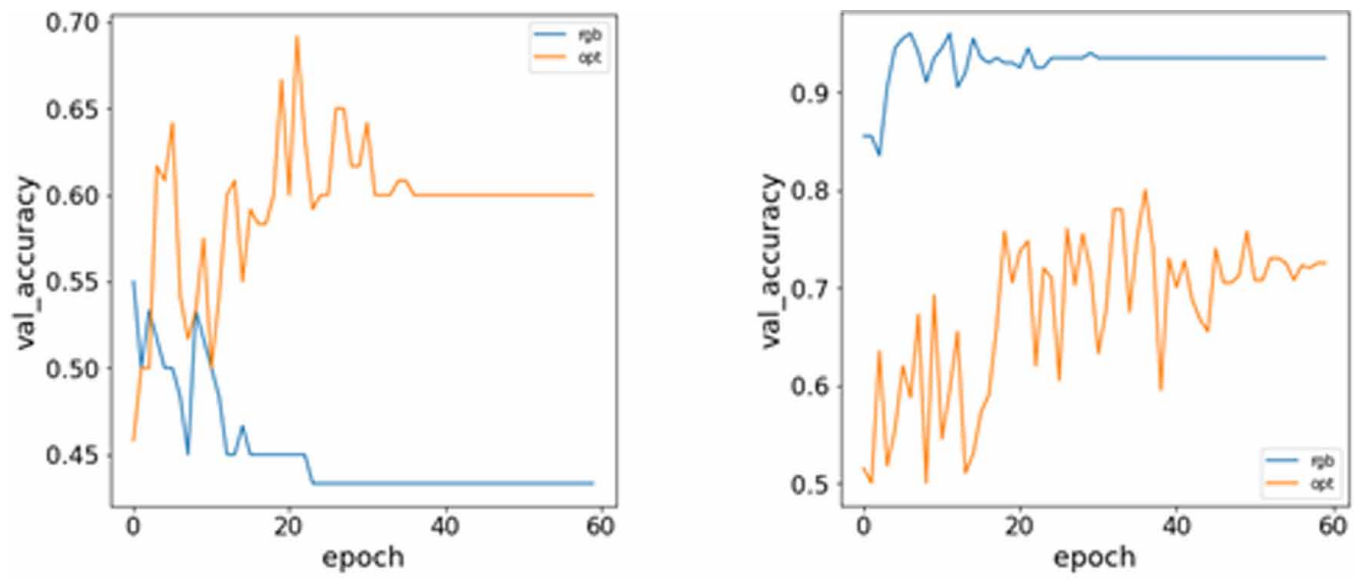

(a) Comparison on the Surveillance dataset. (b) Comparison on the Hockey dataset.

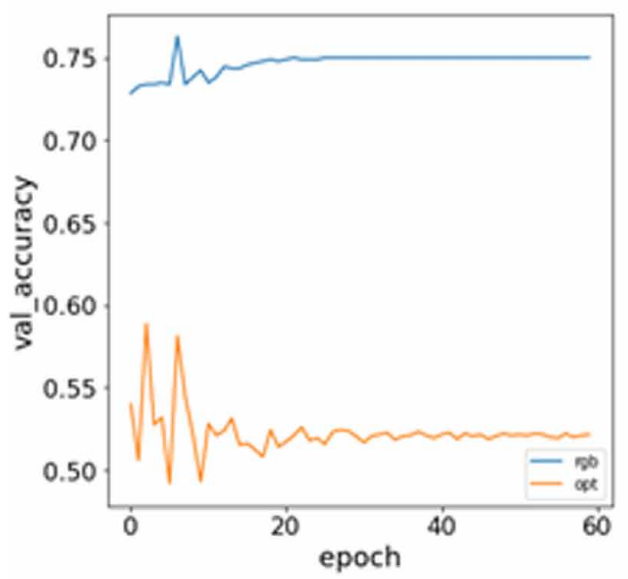

(c) Comparison on the BL-2314 dataset.

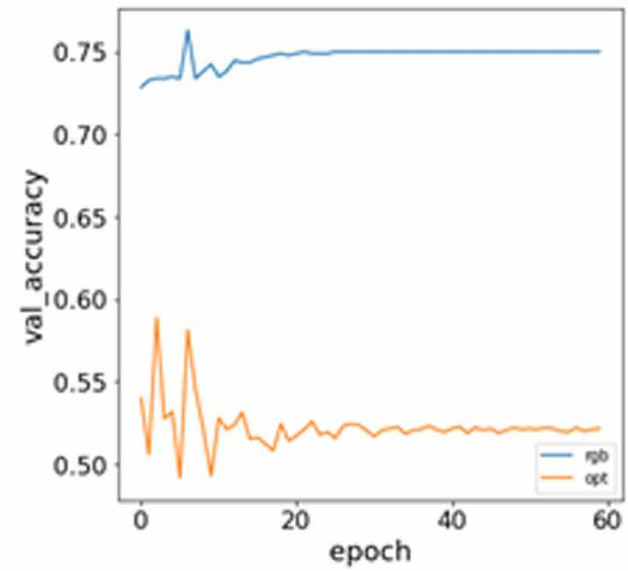

(d) Comparison on the HK-10000 dataset.

sensitivity to the train-test ratio of dataset and the effect of RGB stream and optical stream, are used to insight the performance and characteristics of C3D two-stream network. Through experiments the authors can obtain the following three characteristics of the C3D two-stream network:

1. Compared with other deep learning models, the C3D two-stream network performs better on small-scale datasets.

2. C3D two-stream network on different datasets are not equally sensitive to the train-test ratio.

3. RGB stream have positive feedback for large-scale datasets, while optical stream are positive for small-scale datasets. Therefore optical flow and RGB flow have different desires for the amount of data, and excessive data will degrade the performance of the model. 
Of course, authors' exploration of the model is not comprehensive enough, for example, the authors have not discussed the impact of multiple scenes and single scenes on the model in the datasets, and authors' next study will explore this issue. In the future, in addition to studying the above issue, the authors will try to apply violence detection in combination with target detection on surveillance cameras.

\section{ACKNOWLEDGMENT}

This work was supported by the National Natural Science Foundation of China (Nos. 61663009, 61762036, 61702236), the Science and Technology Plan Projects of Zhangzhou (Nos. ZZ2020J06, ZZ2020J24), the Natural Science Foundation of Jiangxi Province (No. GJJ200629), and the President's Fund of Minnan Normal University (No. MS001). 


\section{REFERENCES}

Accattoli, S., Sernani, P., Falcionelli, N., Mekuria, D. N., \& Dragoni, A. F. (2020). Violence detection in videos by combining 3D convolutional neural networks and support vector machines. Applied Artificial Intelligence, 34(4), 329-344. doi:10.1080/08839514.2020.1723876

Aktı, S.., Tataroğlu, G. A., \& Ekenel, H. K. (2019, November). Vision-based fight detection from surveillance cameras. In 2019 Ninth International Conference on Image Processing Theory, Tools and Applications (IPTA) (pp. 1-6). IEEE.

Breiman, L. (2001). Random forests. Machine Learning, 45(1), 5-32. doi:10.1023/A:1010933404324

Carreira, J., \& Zisserman, A. (2017). Quo vadis, action recognition? a new model and the kinetics dataset. In Proceedings of the IEEE Conference on Computer Vision and Pattern Recognition (pp. 6299-6308). doi:10.1109/ CVPR.2017.502

Chen, L. H., Hsu, H. W., Wang, L. Y., \& Su, C. W. (2011, August). Violence detection in movies. In 2011 Eighth International Conference Computer Graphics, Imaging and Visualization (pp. 119-124). IEEE. doi:10.1109/ CGIV.2011.14

Clarin, C., Dionisio, J., Echavez, M., \& Naval, P. (2005). DOVE: Detection of movie violence using motion intensity analysis on skin and blood. PCSC, 6, 150-156.

Cortes, C., \& Vapnik, V. (1995). Support-vector networks. Machine Learning, 20(3), 273-297. doi:10.1007/ BF00994018

Dalal, N., \& Triggs, B. (2005, June). Histograms of oriented gradients for human detection. In 2005 IEEE Computer Society Conference on Computer Vision and Pattern Recognition (CVPR'05) (Vol. 1, pp. 886-893). IEEE. doi:10.1109/CVPR.2005.177

Ding, C., Fan, S., Zhu, M., Feng, W., \& Jia, B. (2014, December). Violence detection in video by using 3D convolutional neural networks. In International Symposium on Visual Computing (pp. 551-558). Springer. doi:10.1007/978-3-319-14364-4_53

Donahue, J., Anne Hendricks, L., Guadarrama, S., Rohrbach, M., Venugopalan, S., Saenko, K., \& Darrell, T. (2015). Long-term recurrent convolutional networks for visual recognition and description. In Proceedings of the IEEE Conference on Computer Vision and Pattern Recognition (pp. 2625-2634). doi:10.1109/ CVPR.2015.7298878

Febin, I. P., Jayasree, K., \& Joy, P. T. (2019). Violence detection in videos for an intelligent surveillance system using MoBSIFT and movement filtering algorithm. Pattern Analysis \& Applications, 1-13.

Feichtenhofer, C., Pinz, A., \& Zisserman, A. (2016). Convolutional two-stream network fusion for video action recognition. In Proceedings of the IEEE Conference on Computer Vision and Pattern Recognition (pp. 19331941). doi:10.1109/CVPR.2016.213

Gao, Y., Liu, H., Sun, X., Wang, C., \& Liu, Y. (2016). Violence detection using oriented violent flows. Image and Vision Computing, 48, 37-41. doi:10.1016/j.imavis.2016.01.006

Hassner, T., Itcher, Y., \& Kliper-Gross, O. (2012, June). Violent flows: Real-time detection of violent crowd behavior. In 2012 IEEE Computer Society Conference on Computer Vision and Pattern Recognition Workshops (pp. 1-6). IEEE.

He, K., Zhang, X., Ren, S., \& Sun, J. (2016). Deep residual learning for image recognition. In Proceedings of the IEEE Conference on Computer Vision and Pattern Recognition (pp. 770-778). IEEE.

Huang, F., Zhang, X., Zhao, Z., Xu, J., \& Li, Z. (2019). Image-text sentiment analysis via deep multimodal attentive fusion. Knowledge-Based Systems, 167, 26-37. doi:10.1016/j.knosys.2019.01.019

Ji, S., Xu, W., Yang, M., \& Yu, K. (2012). 3D convolutional neural networks for human action recognition. IEEE Transactions on Pattern Analysis and Machine Intelligence, 35(1), 221-231. doi:10.1109/TPAMI.2012.59 PMID:22392705

LeCun, Y., Bottou, L., Bengio, Y., \& Haffner, P. (1998). Gradient-based learning applied to document recognition. Proceedings of the IEEE, 86(11), 2278-2324. doi:10.1109/5.726791 
Lowe, D. G. (2004). Distinctive image features from scale-invariant keypoints. International Journal of Computer Vision, 60(2), 91-110. doi:10.1023/B:VISI.0000029664.99615.94

Mahadevan, V., Li, W., Bhalodia, V., \& Vasconcelos, N. (2010, June). Anomaly detection in crowded scenes. In 2010 IEEE Computer Society Conference on Computer Vision and Pattern Recognition (pp. 1975-1981). IEEE.

Nievas, E. B., Suarez, O. D., Garcia, G. B., \& Sukthankar, R. (2011). Hockey fight detection dataset. In Computer Analysis of Images and Patterns (pp. 332-339). Springer.

Ramzan, M., Abid, A., Khan, H. U., Awan, S. M., Ismail, A., Ahmed, M., Ilyas, M., \& Mahmood, A. (2019). A review on state-of-the-art violence detection techniques. IEEE Access: Practical Innovations, Open Solutions, 7, 107560-107575. doi:10.1109/ACCESS.2019.2932114

Roshan, S., Srivathsan, G., Deepak, K., \& Chandrakala, S. (2020). Violence detection in automated video surveillance: recent trends and comparative studies. The Cognitive Approach in Cloud Computing and Internet of Things Technologies for Surveillance Tracking Systems, 157-171.

Russakovsky, O., Deng, J., Su, H., Krause, J., Satheesh, S., Ma, S., Huang, Z., Karpathy, A., Khosla, A., Bernstein, M., Berg, A. C., \& Fei-Fei, L. (2015). Imagenet large scale visual recognition challenge. International Journal of Computer Vision, 115(3), 211-252. doi:10.1007/s11263-015-0816-y

Si, C., Chen, W., Wang, W., Wang, L., \& Tan, T. (2019). An attention enhanced graph convolutional lstm network for skeleton-based action recognition. In Proceedings of the IEEE/CVF Conference on Computer Vision and Pattern Recognition (pp. 1227-1236). doi:10.1109/CVPR.2019.00132

Simonyan, K., \& Zisserman, A. (2014). Two-stream convolutional networks for action recognition in videos. arXiv preprint arXiv:1406.2199.

Singh, A., Patil, D., \& Omkar, S. N. (2018). Eye in the sky: Real-time drone surveillance system (DSS) for violent individuals identification using ScatterNet hybrid deep dearning network. In Proceedings of the IEEE Conference on Computer Vision and Pattern Recognition Workshops (pp. 1629-1637). doi:10.1109/CVPRW.2018.00214

Song, W., Zhang, D., Zhao, X., Yu, J., Zheng, R., \& Wang, A. (2019). A novel violent video detection scheme based on modified 3D convolutional neural networks. IEEE Access: Practical Innovations, Open Solutions, 7, 39172-39179. doi:10.1109/ACCESS.2019.2906275

Tran, D., Bourdev, L., Fergus, R., Torresani, L., \& Paluri, M. (2015). Learning spatiotemporal features with 3d convolutional networks. In Proceedings of the IEEE International Conference on Computer Vision (pp. 44894497). doi:10.1109/ICCV.2015.510

Ullah, F. U. M., Ullah, A., Muhammad, K., Haq, I. U., \& Baik, S. W. (2019). Violence detection using spatiotemporal features with 3D convolutional neural network. Sensors (Basel), 19(11), 2472. doi:10.3390/ s19112472 PMID:31151184

Wang, H., \& Schmid, C. (2013). Action recognition with improved trajectories. In Proceedings of the IEEE International Conference on Computer Vision (pp. 3551-3558). IEEE.

Wu, P., Liu, J., Shi, Y., Sun, Y., Shao, F., Wu, Z., \& Yang, Z. (2020, August). Not only look, but also listen: Learning multimodal violence detection under weak supervision. In European Conference on Computer Vision (pp. 322-339). Springer.

Xia, Q., Zhang, P., Wang, J., Tian, M., \& Fei, C. (2018, August). Real time violence detection based on deep spatio-temporal features. In Chinese Conference on Biometric Recognition (pp. 157-165). Springer. doi:10.1007/978-3-319-97909-0_17

Yan, S., Xiong, Y., \& Lin, D. (2018, April). Spatial temporal graph convolutional networks for skeleton-based action recognition. Proceedings of the AAAI Conference on Artificial Intelligence, 32(1).

Zhou, P., Ding, Q., Luo, H., \& Hou, X. (2018). Violence detection in surveillance video using low-level features. PLoS One, 13(10), e0203668. doi:10.1371/journal.pone.0203668 PMID:30281588 


\section{ENDNOTES}

1 The two constructed datasets are made publicly available https://pan.baidu.com/s/1aULb01-ueH9FRt9oUtF1g, and the download code is mnnu.

https://haokan.baidu.com/

https://www.bilibili.com/

Zanzan Lu is now studying in the School of Computer Science, Minnan Normal University, with a professional approach to computer vision.

Xuewen Xia is now teaching in the School of Physics and Information in Minnan Normal University, a graduate advisor, and his professional direction is computational intelligence and its applications.

Hongrun Wu received the Ph.D. degree from Wuhan University, Wuhan, China, in 2018. She is currently an associate professor with the School of Physics and Information Engineering, Minnan Normal University, Zhangzhou, China. Her current research interests include computational intelligence and its applications in the field of graph neural networks and computer vision.

Chen Yang received the Ph.D. degree in software engineering from both the University of Groningen, Groningen, The Netherlands, and Wuhan University, Wuhan, China, in 2018. He has worked for IBO Technology Co., Ltd. from June 2018 to May 2021. He is currently a senior researcher in the School of Artificial Intelligence at Shenzhen Polytechnic, China. His research interests concern the area of software engineering and artificial intelligence. 